\title{
Una Estrategia para el Aprendizaje de la Cultura Científica
}

\author{
Luz M. González ${ }^{(1)}$ y Margarita Rasilla ${ }^{(2)}$ \\ (1) Escuela Superior de Física y Matemáticas del IPN. Edificio 9 U.P. Adolfo López Mateos, \\ Col. San Pedro Zacatenco, C.P. 07730, México, D.F. - México (e-mail: luzmar@esfm.ipn.mx) \\ (2) Centro Interdisciplinario de Investigación para el Desarrollo Integral Regional del IPN unidad \\ Oaxaca, Hornos no. 1003, col. Noche buena, C.P. 71230 Santa Cruz Xoxocotlan, Oaxaca-México \\ (e-mail: mrasilla@hotmail.com)
}

Recibido Ene. 06, 2011; Aceptado Feb. 08, 2011; Versión final recibida Feb. 28, 2011

\begin{abstract}
Resumen
El objetivo de este trabajo es identificar las necesidades formativas de los estudiantes de licenciatura en Física y Matemáticas, de la Escuela Superior de Física y Matemáticas del Instituto Politécnico Nacional en México, para el aprendizaje de la cultura científica. Para ello se utiliza como eje la competencia investigativa. El artículo parte con una reflexión acerca de lo que es la cultura en general, y lo que es la cultura científica en particular. Luego se presentan los resultados de una investigación de corte cualitativo, realizada para identificar las necesidades. Se propone integrar elementos de la divulgación científica al inicio de las unidades didácticas, para ir avanzando en la formalización, mediante el uso de contextos cada vez más lejanos a la experiencia de los estudiantes. El artículo muestra que el diseño de estrategias centradas en el logro de competencias específicas es una alternativa válida para contribuir a la apropiación de la cultura científica
\end{abstract}

Palabras clave: cultura científica, divulgación científica, educación formal, enseñanza-aprendizaje

\section{A Strategy for Learning Scientific Culture}

\begin{abstract}
The aim of this study is to identify the formative needs of physics and mathematics students of the Escuela Superior de Física y Matemática of the Insituto Politécnico Nacional in Mexico, for the better learning and understanding of scientific culture. Research competence has been used as the main axis of the study. The article begins with a reflection on culture in general and scientific culture in particular. After that, the results of a qualitative research project performed to identify the formative needs are presented. It is proposed to integrate the elements of scientific dissemination at the beginning of teaching units, to the make progress on the study, through the use of contexts which are increasingly more distant from students' experience. The article shows that the design of strategies centered on the development of specific competencies is a valid option for facilitating students' absorption of scientific culture.
\end{abstract}




\section{INTRODUCCIÓN}

En el diccionario de la Real Academia Española, en su XXII edición, se define cultura primero como: "conjunto de conocimientos que permite a alguien desarrollar su juicio crítico", y en seguida como "conjunto de modos de vida y costumbres, conocimientos y grado de desarrollo artístico, científico, industrial, en una época, grupo social, etc". Para Eco (1986), la cultura consiste en establecer qué y cómo una función de un objeto puede repetirse y transmitir esta información a alguien, que puede ser uno mismo, pasado un tiempo. Entonces, para que surja cultura, se requiere que un ser pensante establezca una nueva función de un objeto; la denomine y reconozca la relación del objeto con dicha función.

"Digamos que surge la cultura cuando (y no sabemos si el australopiteco se encuentra en estas condiciones): a) un ser pensante establece una nueva función de la piedra (no es necesario pulirla para convertirla en buril); b) lo «denomina» «piedra que sirve para algo» (no es necesario denominarla en alta voz o comunicarlo a los demás); c) la reconoce como «la piedra que corresponde a la función $X$ y que tiene el nombre $Y$ » (tampoco hace falta denominarla una segunda vez: basta con reconocerlo). Utilizar una piedra por primera vez no es cultura. Establecer qué y 2 cómo la función puede repetirse y transmitir esta información del náufrago solitario de hoy al náufrago solitario de mañana, esto sí lo es" (ibídem).

Así, se tiene que existe cultura cuando se establece la definición (qué) y la técnica (cómo) acerca de la repetición de una acción útil (para qué); se tiene la capacidad de transmitir dicha información (a quiénes); y se desarrolla el juicio crítico (qué aporta). Las palabras entre paréntesis, pretenden ilustrar las preguntas a las cuales se respondería con la creación de un elemento de la cultura, y si se comparan éstas con las preguntas que utiliza la didáctica para enseñar significativamente se observa una similitud (Sanmartí, 2002). Ésta se debe a que la educación busca promover que la cultura generada a través de los siglos pueda ser comprendida por la población, se sepa aplicar y se pueda continuar generando. La función de la educación es, entonces, entregar la cultura a las nuevas generaciones, pero no como un cúmulo de ideas, sino como una propuesta susceptible de ser modificada, enriquecida con oportunidades para que aprendan a trabajar para generar dichas modificaciones.

Una estrategia que se está utilizando para lograr que los estudiantes puedan realizar dicha aplicación, es optar por modelos basados en competencias. Sin embargo, Abrate y otros (2007), observaron que "en niveles superiores los estudiantes tienen problemas para transferir el conocimiento a los diferentes contextos en que se aplican". Esta conclusión se obtuvo con estudiantes de ciencias económicas, en un ambiente en el cual "los procesos de enseñanza de la Matemática se encuentran intensamente guiados por los profesores, basados tal vez en la creencia de que el alumno aprende viendo y el docente enseña mostrando (ibídem). Entonces surge la pregunta ¿los estudiantes que ingresan al área de ciencias físico matemáticas de ese mismo nivel, son capaces de aplicar el conocimiento en diferentes contextos? Por lo que fue del interés de este grupo dar respuesta a esta interrogante. Para delimitar el problema, la investigación se centró en evaluar la aplicación de temas de ciencias físico matemáticas; por lo que el objeto a evaluar, corresponde a la "cultura científica", la cual presupone la incorporación de los conocimientos e innovaciones científicas en los individuos y en la sociedad.; No solamente implica el acceso a una información especializada, sino también la elaboración de percepciones y opiniones, que conforman la posibilidad de sustentar un juicio crítico frente a la ciencia y la tecnología. El acceso a la información, la integración de los conocimientos y el desarrollo de aptitudes para la acción constituyen el núcleo de la cultura científica (Sebastian, 2006).

La educación científica se debe concebir como un proceso de inmersión en las formas propias de proceder del ambiente científico, a la manera como el aprendiz de artista va siendo imbuido, como por ósmosis, en la forma peculiar de ver las cosas característica de la escuela en la que se entronca". Sin embargo, la educación científica formal que se imparte en las escuelas de los diferentes niveles educativos, generalmente se realiza mediante procesos muy diferentes a la mencionada, por lo que no se favorece de manera intencionada que los estudiantes tengan oportunidades de realizar dicha inmersión, generando un proceso que genera aversión en muchos 
de los casos. En la Tabla 1, se presentan algunas características de la ciencia y las implicaciones didácticas de las mismas, que muestran oportunidades que pueden aprovecharse para cultivar la cultura científica en la educación formal (Gil y Guzmán 1993).

Tabla 1: Algunas características de la ciencia y sus implicaciones

\begin{tabular}{|c|c|c|}
\hline La Ciencia & Implicación Didáctica & $\begin{array}{c}\text { Justificación } \\
\end{array}$ \\
\hline $\begin{array}{l}\text { Es empírica en su } \\
\text { intervención. }\end{array}$ & $\begin{array}{l}\text { Cuidar y cultivar la intuición en } \\
\text { general, la manipulación } \\
\text { operativa del espacio y de los } \\
\text { símbolos. }\end{array}$ & $\begin{array}{l}\text { Si se tiene en cuenta la experiencia, la } \\
\text { manipulación y las aplicaciones, se } \\
\text { hacen patentes la fecundidad y potencia } \\
\text { de la ciencia. }\end{array}$ \\
\hline $\begin{array}{l}\text { Sus resultados poseen un } \\
\text { carácter formal. }\end{array}$ & $\begin{array}{l}\text { Promover la comprensión e } \\
\text { inteligencia de lo que se hace, } \\
\text { después de la intuición. }\end{array}$ & $\begin{array}{l}\text { A cada fase de desarrollo mental, como a } \\
\text { cada etapa histórica o a cada nivel } \\
\text { científico, le corresponde su propio rigor. }\end{array}$ \\
\hline $\begin{array}{l}\text { Evoluciona por medio de } \\
\text { experimentos, y de } \\
\text { tentativas sucesivas, } \\
\text { fructuosas y estériles. }\end{array}$ & $\begin{array}{l}\text { Acudir a la propia historia de la } \\
\text { ciencia, revela ese proceso de } \\
\text { emergencia del conocimiento en } \\
\text { el tiempo. }\end{array}$ & $\begin{array}{l}\text { La enseñanza ideal debería tratar de } \\
\text { reflejar este carácter profundamente } \\
\text { humano de la ciencia, ganando con ello } \\
\text { en asequibilidad, dinamismo e interés. }\end{array}$ \\
\hline $\begin{array}{l}\text { El método predomina sobre } \\
\text { el contenido (saber hacer). }\end{array}$ & $\begin{array}{l}\text { Enfatizar más en los procesos } \\
\text { de pensamiento propios de la } \\
\text { ciencia. }\end{array}$ & $\begin{array}{l}\text { Los procesos verdaderamente eficaces } \\
\text { de pensamiento no se vuelven obsoletos } \\
\text { con tanta rapidez como los contenidos. }\end{array}$ \\
\hline $\begin{array}{l}\text { Los verdaderos problemas } \\
\text { científicos suelen implicar } \\
\text { trabajo rutinario. }\end{array}$ & $\begin{array}{l}\text { Enfatizar más en la comprensión } \\
\text { de los procesos que en la } \\
\text { ejecución de rutinas. }\end{array}$ & $\begin{array}{l}\text { Usando tecnología adecuada, se evita el } \\
\text { sentimiento de esterilidad del tiempo } \\
\text { empleado, que se genera por la } \\
\text { repetición de lo que ya se domina. }\end{array}$ \\
\hline $\begin{array}{l}\text { La historia, los desarrollos } \\
\text { de la sociedad, los } \\
\text { elementos afectivos y la } \\
\text { ciencia, se impactan } \\
\text { mutuamente. }\end{array}$ & $\begin{array}{l}\text { Favorecer que se perciba el } \\
\text { sentimiento estético y lúdico de } \\
\text { la ciencia, a fin de involucrarlos } \\
\text { en ella de un modo más } \\
\text { personal y humano. }\end{array}$ & $\begin{array}{l}\text { Reduce el posicionamiento inicial } \\
\text { afectivo destructivo de sus propias } \\
\text { potencialidades en este campo, que es } \\
\text { provocado, en muchos casos, por una } \\
\text { introducción inadecuada. }\end{array}$ \\
\hline
\end{tabular}

Si se analizan las implicaciones didácticas mencionadas, se puede observar que algunas tienen carácter de divulgación científica. Es importante mencionar que los primeros niveles de formación, se diseñan de tal manera que el carácter divulgativo de la ciencia es el elemento predominante y gradualmente, en los niveles siguientes, prevalece la educación formal de la ciencia. De la misma manera, cuando se comienza a trabajar un tema que resulta más o menos novedoso para los estudiantes, aún en el nivel superior, conviene comenzar con actividades de carácter divulgativo. Al comparar algunas de las actividades que se usan comúnmente en ambos procesos (divulgación y educación formal), se puede observar que existen diferencias que hacen que la educación formal esté lejana a la experiencia de los estudiantes (tabla 2). Esta distancia genera obstáculos en el aprendizaje para los estudiantes.

Tabla 2: La educación formal y la divulgación científica

\begin{tabular}{|l|l|}
\hline Educación formal & Divulgación \\
\hline Se busca el rigor & Se evita el rigor \\
\hline Veraz y precisa & Veraz pero matizada con hipérbole y uso de metáforas \\
\hline Abstracta & Vinculada con la realidad \\
\hline Lenguaje especializado & Lenguaje coloquial \\
\hline Expositiva y argumentativa & Expositiva,, argumentativa, narrativa, dialógica. \\
\hline Usa dibujos, esquemas y gráficos & Usa todo tipo de recursos \\
\hline
\end{tabular}

Para reducir esa distancia se requieren conocer los elementos de la cultura de los estudiantes, con el objetivo de elaborar un plan de inmersión de los mismos en la cultura científica, así que se eligió una población, que está constituida por los estudiantes de licenciatura en Física de la Escuela Superior de Física y Matemáticas del Instituto Politécnico Nacional, así como una competencia transversal muy Inclusiva, es decir, que se trabajen elementos de ella en la mayoría de las asignaturas y que refleje lo más significativo de dicha licenciatura, la cual fue la competencia investigativa. Debido a que dicha competencia es muy compleja, se considera 
importante dividirla en varios aspectos que son: Epistemológicos, que aportan una visión acerca de la naturaleza de la ciencia y su método; metodológicos, que tienen que ver con el desarrollo de las habilidades y destrezas que se requieren para la generación de cultura científica; lingüísticos relacionados con el uso del lenguaje de la ciencia; cognitivos los cuales se orientan hacia el uso de los conocimientos al enfrentar problemas y éticos para fomentar una actitud reflexiva acerca del posible impacto de la ciencia y la tecnología que se genere, en la vida, la salud y el entorno en general (Ladino, 2001).

\section{METODOLOGÍA}

Para indagar acerca de los requerimientos de la Licenciatura en Física, con respecto a la competencia investigativa, se realizó una investigación teórica que consistió en la revisión de las competencias propuestas por el proyecto Tuning (Beneitone, 2007), con la participación de los profesores de la Academia de Física Experimental; posteriormente se revisaron propuestas de otras licenciaturas (Programas oficiales, Universidad de Barcelona, y de Almería); y de bachillerato, debido a que es el antecedente inmediato, se utilizaron los proyectos del Bachillerato del Nivel Medio Superior RIEMS de México (SEMS-SEP), y el Proyecto 2061 de Estados Unidos (AAAS, 2009). Para conocer el nivel de desempeño de los estudiantes, con respecto a la competencia investigativa, se procesaron los datos de una investigación experimental de corte cualitativo, con metodología constructivista, la cual se centra en indagar el significado de un fenómeno para los implicados en un programa social, mediante la aplicación de problemas abiertos en contexto (Camarena, 2006), a muestras de 15 estudiantes en promedio, tanto de ingreso reciente en la licenciatura, como a los que están por egresar. Las soluciones que aportaron se analizaron mediante matrices de evaluación y redes sistémicas (González, 2010). En la que se obtuvieron los siguientes resultados:

\section{RESULTADOS}

\section{Aspecto Epistemológico}

El objetivo de este aspecto es favorecer la evolución de las ideas acerca de la naturaleza de la ciencia y su método, que les permita a los estudiantes ver el dinamismo con el que se desarrolla, así como la creatividad que implica la tarea científica y orienta la innovación (Flores et al, 2009) aquí se aplicó un cuestionario semiestructurado (figura 1), el que se validó a través de su aplicación a una muestra estratificada, que se integró desde estudiantes de ingreso reciente, hasta egresados. Las respuestas obtenidas en la validación, permitieron valorar el aspecto epistemológico en cuatro dimensiones, analizados con fundamento en la bibliografía: Los criterios de demarcación que usan para decidir si un conocimiento es científico; las ideas acerca de la metodología científica; los patrones de cambio que se siguen en la generación del conocimiento científico y el estatus que le asignan a dicho conocimiento Para ilustrar la forma como se asimilaron las respuestas, para la elaboración de la red (figura 2), se muestran unas respuestas textuales. Por ejemplo, una en que se afirma que la Astrología si es ciencia.

Lee la siguiente noticia, y responde a las preguntas que se presentan a continuación:

Una encuesta hecha por Gallup en 1984 indicó que el 55\% de los adolescentes americanos creen que la Astrología funciona. Secciones de astrología aparecen en más de 1200 periódicos en los Estados Unidos. $Y$ en todo el mundo, la gente basa sus decisiones personales, financieras y hasta médicas, en el consejo de los astrólogos...

Algunos astrólogos mantienen que podría haber una fuerza aún desconocida que representa la influencia astrológica (Cfr.http://www.periodicotribuna.com.ar/Articulo.asp ?Articulo=1378).

1. ¿Es la astrología conocimiento científico? Explica tu respuesta.

2. llustra mediante un esquema, cómo se elabora el conocimiento científico.

3. Mediante un ejemplo, explica cómo se hace la ciencia.

Fig. 1: Cuestionario para evaluar el aspecto epistemológico 


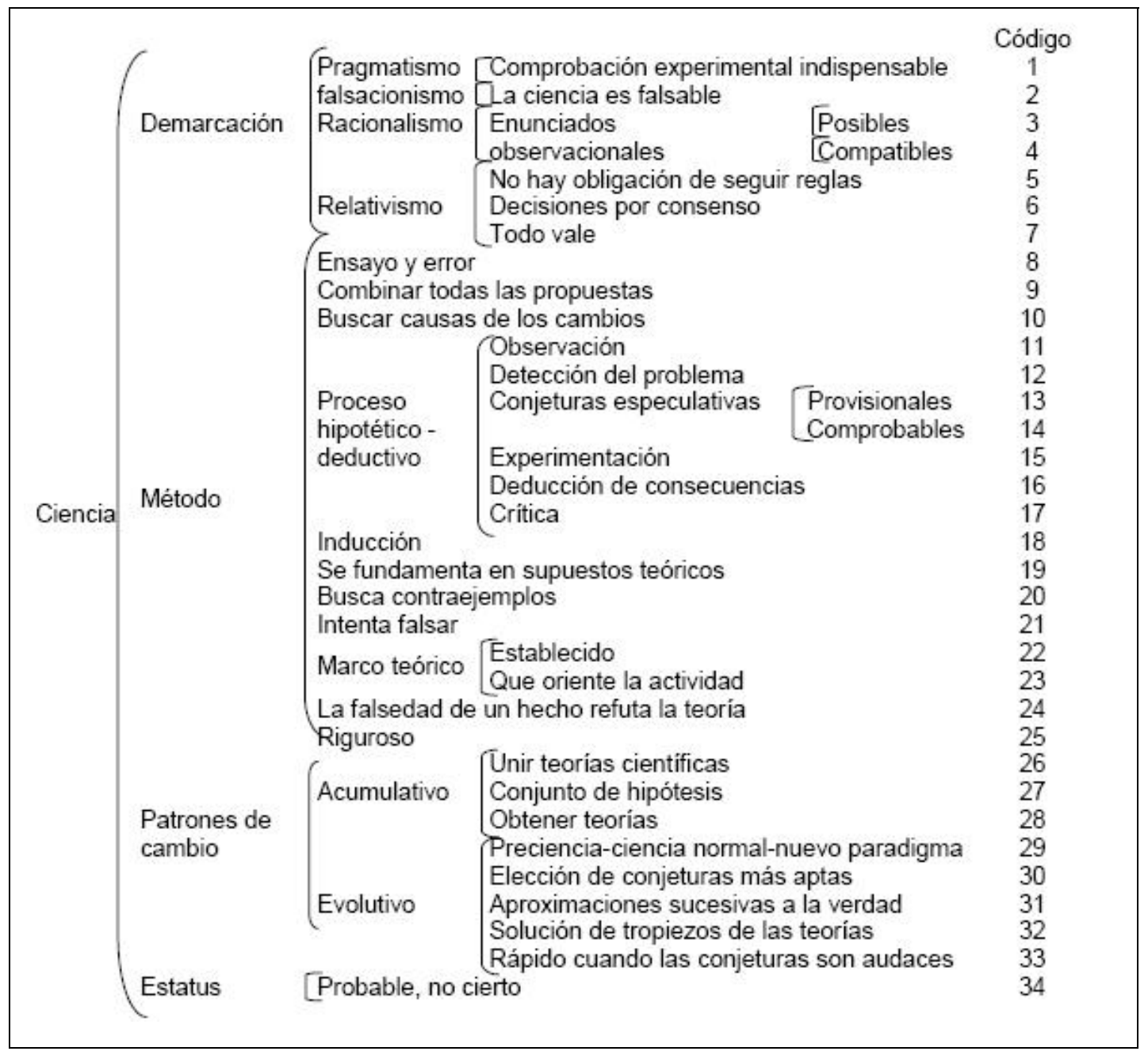

Fig. 2: Red sistémica de la validación del cuestionario para evaluar el aspecto epistemológico de la competencia investigativa

La ciencia también parte de postulados que se toman por fe. La carta astral se fundamenta matemáticamente, está basada en estudios probabilísticos, somos partículas elementales (cargas, campos) que interactúan con las demás. La relación de las ideas expresadas en el ejemplo, con las categorías de la red sistémica de la figura 2 son: i) La ciencia también parte de postulados que se toman por fe.- Relativismo, no hay obligación de seguir reglas (código No. 5); ii) La carta astral se fundamenta matemáticamente, está basada en estudios probabilísticos.- Estatus, la ciencia es probable, no cierta (código 34); y iii) somos partículas elementales (cargas, campos, etc.) que interactúan con las demás. Método, se trata de buscar causas en los cambios (código 10).

\section{Aspecto metodológico}

Se evaluó el trabajo experimental realizado por los estudiantes, a partir del cual se construyó la red sistémica que se muestra en la figura 3, mediante criterios construidos por las autoras, a partir de su experiencia docente (Nolasco \& González, 2010). Para ilustrar cómo se asimilaron las ideas de los estudiantes para evaluar el uso comprensivo de los conceptos, se presenta el siguiente ejemplo:

Al aplicar una fuerza en un cuerpo para que tenga movimiento es saber cuánta fuerza aplicar en el objeto y qué tan rápida es la reacción al alcanzar la canica.Si se aplica mayor fuerza en la canica será mayor la velocidad y si su fuerza aplicada es menor la velocidad será mínima. En este caso se puede observar que se usan los términos "aceleración" y "velocidad" indistintamente, por lo que se ubica en el código 2 de la red sistémica elaborada que se presenta en la figura 3. Las 
demás categorías se evaluaron durante el desarrollo del trabajo experimental, mediante observación participante. Por ejemplo, para evaluar los procedimientos experimentales, en el código 9 (Uso de instrumentos de medición), se observó que se ajustara el cero de la escala, se utilizara el instrumento de acuerdo con las instrucciones del manual y se realizara la lectura utilizando cifras significativas, expresando el resultado en las unidades correspondientes.

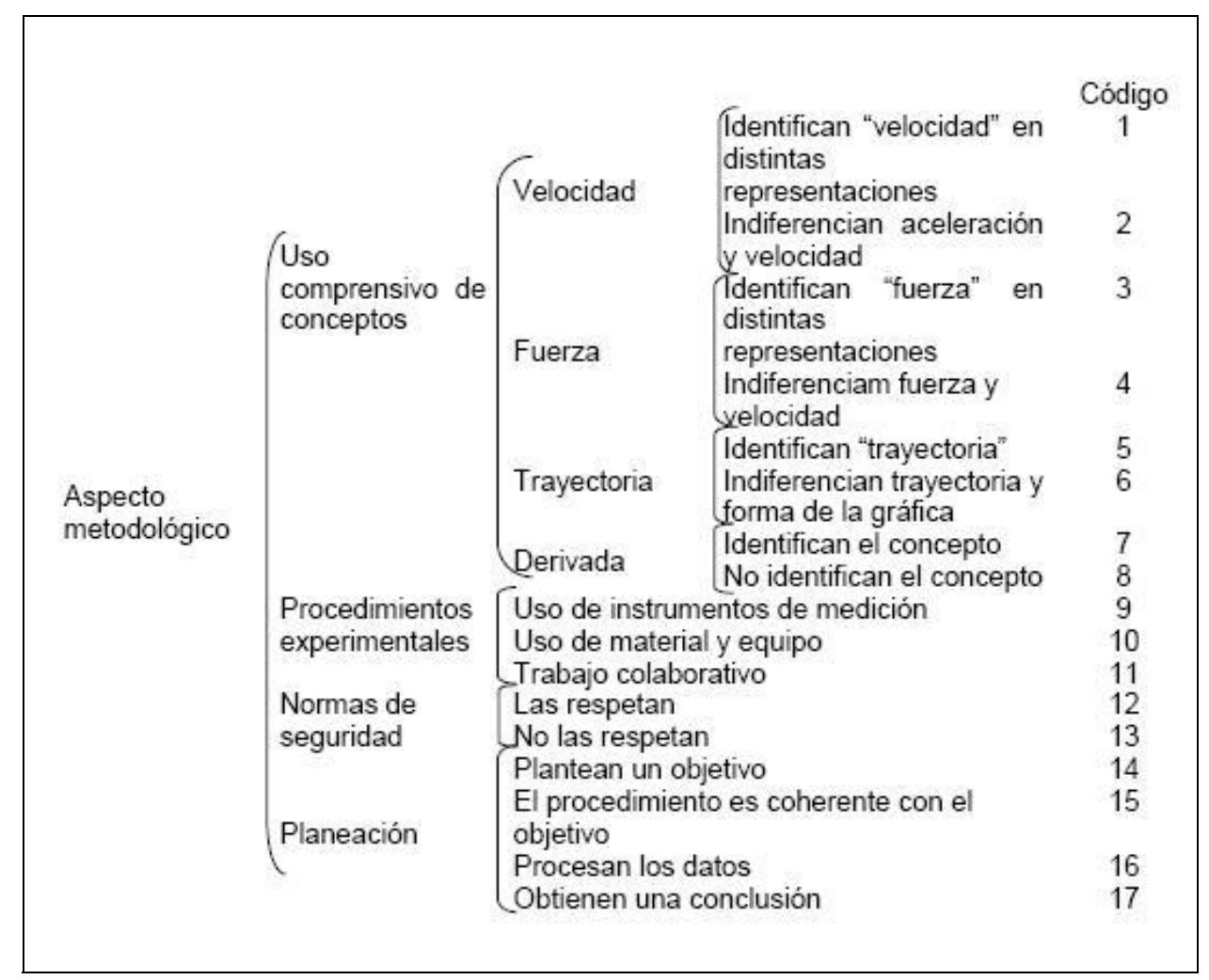

Fig. 3: Red sistémica de la validación de la revisión del trabajo experimental para evaluar el aspecto metodológico de la competencia investigativa.

\section{Aspecto lingüístico}

El uso del lenguaje de la ciencia es indispensable para participar en congresos y escribir artículos que son evaluados por otros, etc., ya que de alguna manera, la actividad científica es una actividad lingüística (Sanmartí, 2003, pp. 138 - 140). Se evaluaron informes de laboratorio, a partir de los cuales se construyó la red sistémica que se muestra en la figura 4, mediante criterios tomados de la bibliografía (Jorba et al, 2000). La canica primero se encuentra en reposo, cuando se le aplicó una fuerza, la canica empezó a rodar en dirección hacia donde se le aplicó la fuerza, ya en movimiento la canica empezó a detenerse y en unas ocasiones la canica se detuvo por completo y en otras se le tuvo que detener.

El fenómeno anterior ocurre porque al principio que no se mueve la que no tiene una fuerza exterior que lo jale o empuja, ya aplicada una fuerza la canica empieza a rodar en dirección a donde se le aplicó la fuerza comienza a detenerse por la acción de la fuerza de rozamiento que empieza a contrarrestar la primera fuerza. En este caso se puede observar que el estudiante utiliza el lenguaje de la ciencia (código 7); argumenta basado en las ideas de ciencia, puesto que habla de conservación del estado de reposo del objeto en movimiento: al principio que no se mueve la que no tiene una fuerza exterior que lo jale o empuja al principio que no se mueve la que no tiene una fuerza exterior que lo jale o empuja (inercia) y la composición de las fuerzas colineales: comienza a detenerse por la acción de la fuerza de rozamiento que empieza a contrarrestar la primera fuerza (código 8); concluye: El fenómeno anterior ocurre porque... (código 10); pero no redacta de manera clara, por la falta de signos de puntuación, principalmente en el segundo párrafo, y por no usar la forma canónica (sujeto, verbo, complemento) en la construcción de las oraciones. 


\begin{tabular}{|c|c|c|c|c|}
\hline $\begin{array}{l}\text { Aspecto } \\
\text { lingüistico }\end{array}$ & $\begin{array}{l}\text { Búsqueda de } \\
\text { información } \\
\text { Relaciones } \\
\text { conceptuales } \\
\text { Procesamiento } \\
\text { de datos }\end{array}$ & $\begin{array}{l}\text { Copian textua } \\
\text { Consultan var } \\
\text { Identifican las } \\
\text { No identifican } \\
\text { Tnterpretan el } \\
\text { Realizan el al } \\
\text { Argumentos } \\
\text { Ortografia } \\
\text { Relaciones } \\
\text { causa-efecto } \\
\text { Esquemas }\end{array}$ & $\begin{array}{l}\text { una sola fuente } \\
\text { fuentes } \\
\text { aciones entre conceptos } \\
\text { relaciones entre conceptos } \\
\text { ste } \\
\text { itmo sin interpretación } \\
\text { Usan el lenguaje de la } \\
\text { ciencia } \\
\text { Se basan en ideas de } \\
\text { ciencia } \\
\text { Concluyen } \\
\text { Redactan de manera clara } \\
\text { Ortografía } \\
\text { Faltas recurrentes } \\
\text { Las identifican } \\
\text { No las identifican } \\
\text { No los usan } \\
\text { No usan diagramas de } \\
\text { cuerpo libre }\end{array}$ & $\begin{array}{c}\text { Código } \\
1 \\
2 \\
3 \\
4 \\
5 \\
6 \\
7 \\
8 \\
\\
9 \\
10 \\
11 \\
12 \\
13 \\
14 \\
15 \\
16\end{array}$ \\
\hline
\end{tabular}

Fig. 4: Red sistémica de la validación de la revisión de los informes del trabajo experimental para evaluar el aspecto lingüístico de la competencia investigativa.

\section{Aspecto cognitivo}

Se elaboró un cuestionario para evaluar dos aspectos, el cognitivo y el ético, consiste en un problema en contexto (figura 5, abajo), construido con base en la teoría de la matemática en el contexto de las ciencias. Las preguntas $a$ y $b$, se refieren al contenido matemático, que en este caso es el concepto de variación, la pregunta $c$ evalúa los elementos que se toman en cuenta para la toma de decisiones, principalmente en el aspecto de la inteligencia emocional de Mayer y Salovey (20012) y la d, la metacognición en la resolución de problemas, de acuerdo con las ideas de Schöenfeld, citado en De Faria (2006), combinadas con las de Flores et al. (2003).

Sedelmayer, un crítico de arte, al comentar acerca de la arquitectura actual, menciona que al diseñar los edificios se cuida que el ambiente de las oficinas resulte adecuado para el buen funcionamiento y cuidado de las computadoras u otros equipos de alto costo; sin embargo, no siempre estas condiciones son ideales para el ser humano. Por ello se han realizado investigaciones cuyo objetivo es identificar las condiciones ideales para poder realizar un trabajo sedentario de manera saludable y confortable.

En la siguiente figura, se muestra la gráfica de la velocidad media del aire permitida, en función de la temperatura del aire, de manera que no exista turbulencia, para un índice de molestia por corrientes de aire de un $15 \%$ de insatisfechos; aplicable a actividades ligeras, esencialmente sedentarias.

De acuerdo con los datos de la gráfica:

a) ¿Cuánto cambia la velocidad del aire, cuando la temperatura se eleva de 22 a $24^{\circ} \mathrm{C}$ ?

b) ¿Si se está ajustando el equipo, qué tan rápido ha de cambiar el valor de velocidad del aire, cuando la temperatura es de $22^{\circ} \mathrm{C}$, para seguir cumpliendo con la norma marcada por la gráfica?

c) Si tienes que decidir entre salirte de la norma para que el equipo dure más o respetar la norma para que las condiciones del ambiente sean más propicias para las personas ¿qué harías? Toma en cuenta que el equipo es

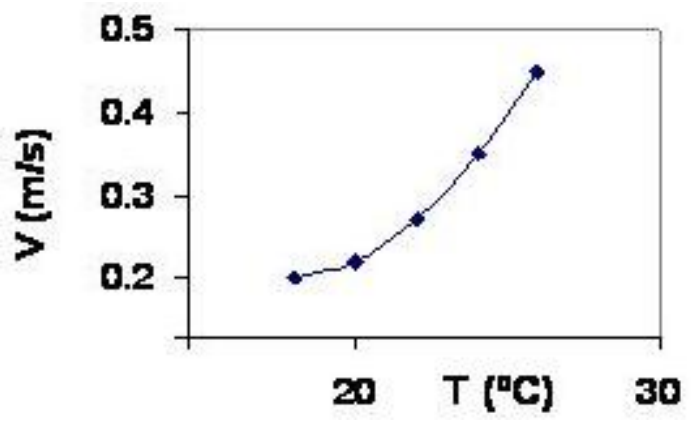
muy costoso. Argumenta tu respuesta y participa en el debate grupal.

d) ¿Cómo respondiste las preguntas a) y b)

e)¿Cómo te sentiste al responderlas?

Fig. 5: Problema en contexto para evaluar el aspecto cognitivo (González y Ruiz, 2009) 
Un ejemplo de respuesta es:

a) Cambia en $8 \mathrm{~m} / \mathrm{s}$.

b) Debe ser un cambio de velocidades al doble en las tres primeras, puesto que la grafica marca $.2, .4, .8$, y en la tercera solo varia $.2 \mathrm{~m} / \mathrm{s} .10$

c) Pues me iría por respetar la norma para que no se afecte a las personas en el ambiente, así se tenga que comprar o reparar el equipo ya sea costoso o no. Argumentando que debemos tener como prioridad al medio ambiente y que si tenemos que utilizar la maquina pues solo debemos ver el beneficio que tenemos y hacer un análisis de cuanto capital es requerido para poder mantener la maquinaria.

d) El a) mmmm... a veces con duda; b) un poco más seguro; c) pues aquí no hubo mucha duda por lo que expuse en el punto anterior. Pero fue más segura mi respuesta.

e) El a), observando cuidadosamente la tabla; el b) observando la posición de los puntos en cada uno de los valores de temperatura o de velocidad; el c) pues fue difícil, pero, los valores que tengo me han enseñado que no debo afectar a terceros en ninguna circunstancia, sino al contrario si es posible ayudar pues hacerlo, es así como me guié para contestar esta última pregunta.

A partir de las respuestas de los estudiantes que resolvieron el problema, se elaboró la red sistémica que se muestra en la figura 6.

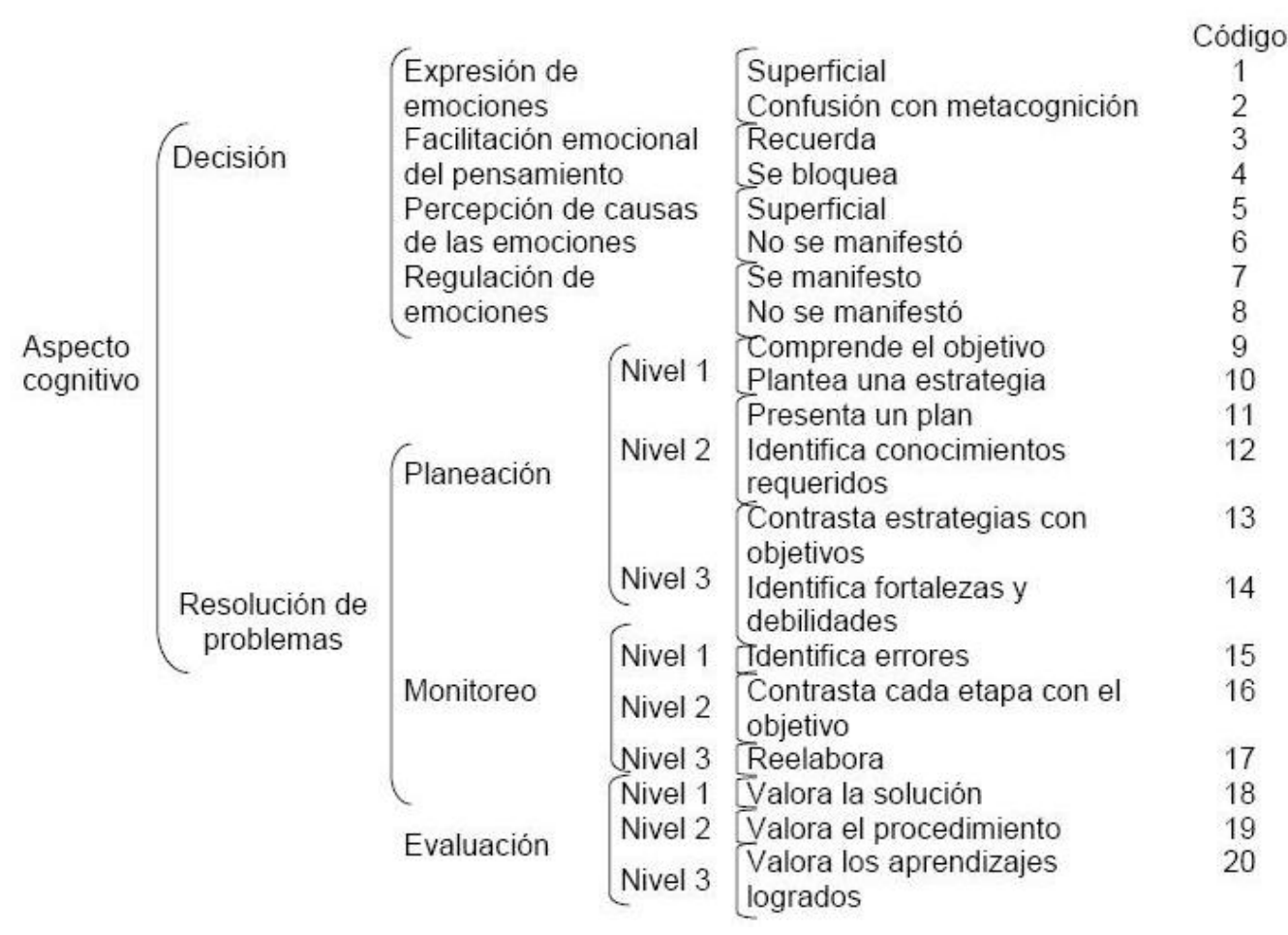

Fig. 6: Red sistémica de la validación del aspecto cognitivo de la competencia investigativa.

De las respuestas del ejemplo se puede observar que en el caso del incremento de la pregunta a, no es del orden esperado, hace falta colocar el punto decimal antes del número 8; en la pregunta b, plantea cómo va cambiando la velocidad, pero lo que se solicitaba era el cambio en un punto, y no lo relacionó con el concepto de derivada. En la pregunta $d$, se puede observar que la identificación de las emociones es superficial (Código 1de la red en la figura 6), se puede inferir un cierto bloqueo (código 4), puesto que en la pregunta a, falló en la colocación del punto decimal. En la pregunta e), se puede ver que comprendió el objetivo de la pregunta $a$, pero no el de la $b$, puesto que lo que hizo no corresponde con la obtención de la rapidez de cambio en un punto (código 9), y no identificó el concepto de derivada (código 12). Como sus respuestas son muy concretas, no se tiene evidencia para los demás códigos de la red. 


\section{Aspecto ético}

La evaluación de este aspecto se realizó mediante la pregunta $c$ del mismo problema que se muestra en la figura 5. El análisis se fundamentó en la teoría cognitiva evolutiva del razonamiento moral (Kolhberg, 1997, pp. 21 - 47), a partir de la cual se elaboró la red sistémica de la figura 7. En la respuesta $c$ del mismo ejemplo que se presenta arriba, se puede interpretar que para decidir le motiva obtener el mayor bien para el mayor número (código 1 de la red sistémica elaborada de la figura 7), que valora más al ser humano que a la empresa, a su trabajo y a los bienes económicos (códigos del 4 al 7) y que respeta a las personas, no las utiliza como un medio (código 4).

Posteriormente se elaboró la tabla 3 para concentrar los datos obtenidos de la muestra. Los criterios utilizados para el análisis son los indicados en la segunda línea de la tabla, en la cual se presentan los totales de los datos para cada aspecto, y en la última fila, la característica fundamental del instrumento de evaluación utilizado. Se expresan los resultados en cantidades relativas, para poder hacer la comparación, puesto que el número de estudiantes de las muestras era diferente.

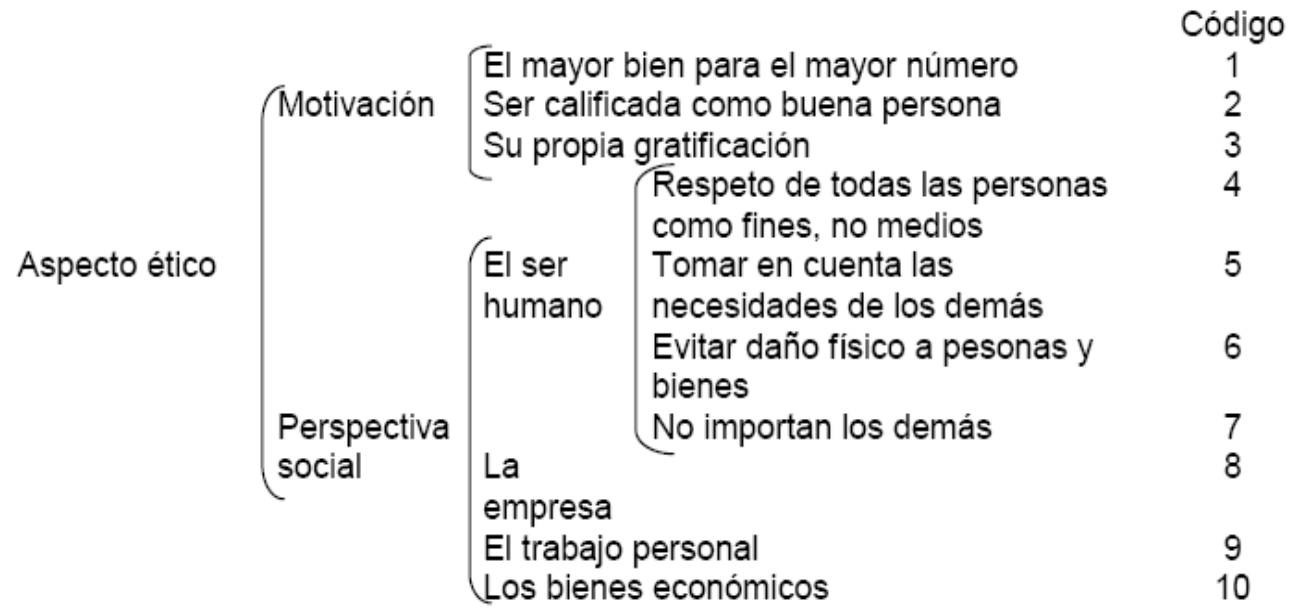

Fig. 7: Red sistémica de la validación del aspecto ético de la competencia investigativa

Tabla 3: Evaluación de los aspectos de la Competencia Investigativa (González y Rasilla, 2010) Estudiante

\begin{tabular}{|c|c|c|c|c|c|c|c|c|c|c|c|c|c|c|c|c|}
\hline \multirow{3}{*}{ Estudiante } & \multicolumn{16}{|c|}{ Evaluación del desempeño de la Competencia Investigativa. Resultados en \% } \\
\hline & \multicolumn{4}{|c|}{ Epistemológicos } & \multicolumn{4}{|c|}{ Metodológicos } & \multicolumn{4}{|c|}{ Lingüísticos } & \multicolumn{2}{|c|}{ Cognitivos } & \multicolumn{2}{|c|}{ Éticos } \\
\hline & a) & b) & c) & d) & a) & b) & c) & d) & a) & b) & c) & d) & & b) & & \\
\hline $\begin{array}{l}\text { Ingreso } \\
\text { reciente }\end{array}$ & 48 & 14 & 0 & 48 & 0 & 39 & 56 & 19 & 13 & 12 & 2 & 3 & 15 & 21 & 5 & 0 \\
\hline Por egresar & 79 & 61 & 11 & 36 & 98 & 57 & 43 & 48 & 38 & 20 & 18 & 18 & 71 & 35 & 14 & 0 \\
\hline $\begin{array}{l}\text { Contenido } \\
\text { del } \\
\text { problema }\end{array}$ & \multicolumn{4}{|c|}{$\begin{array}{l}\text { Diferenciar entre } \\
\text { astrología y } \\
\text { astronomía. }\end{array}$} & \multicolumn{4}{|c|}{$\begin{array}{l}\text { Uso de relaciones } \\
\text { causa-efecto y } \\
\text { análisis de datos } \\
\text { experimentales. }\end{array}$} & \multicolumn{4}{|c|}{$\begin{array}{l}\text { Elección de } \\
\text { información, su uso } \\
\text { y elaboración de } \\
\text { informes. }\end{array}$} & \multicolumn{2}{|c|}{$\begin{array}{l}\text { Obtención de } \\
\text { una razón de } \\
\text { cambio para } \\
\text { tomar } \\
\text { decisiones. }\end{array}$} & \multicolumn{2}{|c|}{$\begin{array}{l}\text { Resolución } \\
\text { de un } \\
\text { dilema } \\
\text { moral. }\end{array}$} \\
\hline \multicolumn{17}{|c|}{$\begin{array}{l}\text { Códigos Epistemológicos a) Criterios de demarcación; b) método científico; c) patrón de cambio; d) } \\
\text { estatus } \\
\text { Metodológicos: a) Uso comprensivo de conceptos; b) procedimientos experimentales; c) normas de } \\
\text { seguridad; d) planeación } \\
\text { Códigos Lingüísticos: a) búsqueda de información; b) relaciones conceptuales; c) procesamiento de datos; } \\
\text { d) elaboración de informes. } \\
\text { Códigos Cognitivos: a) Elementos que intervienen en la toma de decisiones; b) resolución de problemas } \\
\text { Éticos a) motivación; d) perspectiva social. }\end{array}$} \\
\hline
\end{tabular}


De la tabla 3 se puede observar que durante su paso por la licenciatura, los estudiantes de la muestra, en un $98 \%$ han desarrollado el uso comprensivo de los conceptos, correspondiente al aspecto metodológico, pero en los demás aspectos, el desarrollo ha sido menor, principalmente en el ético. Esto se puede explicar si suponemos el hecho de que la docencia se realiza aún, en gran medida, como una clase magistral, en la que los estudiantes realizan pocas actividades de aprendizaje. Esta situación podría estar privilegiando el aprendizaje conceptual, a costa de los otros aprendizajes.

De los datos se pueden observar las siguientes necesidades formativas en los estudiantes: 1. Conceptualización de la ciencia flexible, dinámica y accesible; 2. Desarrollo de las destrezas y habilidades experimentales básicas; 3. Aplicación de sus conocimientos en situaciones reales; y 4. Desarrollo del aspecto lingüístico, en particular del lenguaje de las ciencias.

Ante esta situación, se propone utilizar al comienzo de las unidades didácticas en la educación formal, recursos obtenidos de la divulgación científica. Para ejemplificar la propuesta, se presentan en la Tabla 4 las primeras actividades de una unidad didáctica de un curso de física experimental (diseñadas para atender el aspecto epistemológico de la competencia investigativa) construidas utilizando elementos de la divulgación científica.

Tabla 4: Actividades propuestas para el inicio de la unidad didáctica (González y Rasilla, 2010)

\begin{tabular}{|c|c|c|c|}
\hline Intención & Indicadores & Actividad & Escenario \\
\hline \multirow[b]{2}{*}{$\begin{array}{l}\text { Romper el hielo } \\
\text { (identificar } \\
\text { preconceptos) }\end{array}$} & $\begin{array}{l}\text { Distingue las } \\
\text { características } \\
\text { esenciales de la } \\
\text { ciencia. }\end{array}$ & ¿Astrología o astronomía? & \multirow{2}{*}{$\begin{array}{l}\text { Cuestionario } \\
\text { impreso. (el que se } \\
\text { muestra en la } \\
\text { figura } 1 \text {, en el } \\
\text { aspecto } \\
\text { epistemológico }\end{array}$} \\
\hline & $\begin{array}{l}\text { Identifica a las } \\
\text { aportaciones } \\
\text { experimentales como } \\
\text { modelos que explican } \\
\text { las regularidades de la } \\
\text { Naturaleza. }\end{array}$ & Socialización de las respuestas & \\
\hline $\begin{array}{l}\text { Motivación inicial: } \\
\text { Evidenciar las } \\
\text { ideas de los } \\
\text { estudiantes } \\
\text { acerca de lo que } \\
\text { es y lo que no es } \\
\text { ciencia. }\end{array}$ & $\begin{array}{l}\text { Diferencia las } \\
\text { hipótesis } \\
\text { observacionales de las } \\
\text { especulativas. }\end{array}$ & $\begin{array}{l}\text { Ver el video, guiados por las preguntas: } \\
\text { ¿Qué diferencias entre astrología y } \\
\text { astronomía?. ¿Qué cualidades del } \\
\text { Universo permiten que se construyan } \\
\text { leyes naturales? } \\
\text { ¿A qué le llama "modelos"? ¿Por qué } \\
\text { son diferentes los modelos generados } \\
\text { en diferentes lugares, para la misma } \\
\text { situación? } \\
\text { ¿Qué significan las palabras: } \\
\text { regularidad, permanencia y } \\
\text { previsibilidad? } \\
\text { ¿Por qué Kepler necesitaba a Tycho } \\
\text { Brahe y también Tycho Brahe } \\
\text { necesitaba a Kepler? } \\
\text { ¿Qué dificultades se presentan ante una } \\
\text { idea revolucionaria? } \\
\text { ¿Qué cualidades del investigador } \\
\text { posibilitan la creación de una idea } \\
\text { revolucionaria? }\end{array}$ & $\begin{array}{l}\text { Video "La armonía } \\
\text { de los mundos" } \\
\text { (duración: } 55 \\
\text { minutos) }\end{array}$ \\
\hline \multirow{3}{*}{$\begin{array}{l}\text { Evidenciar las } \\
\text { ideas de los } \\
\text { estudiantes } \\
\text { acerca de lo que } \\
\text { es y lo que no es } \\
\text { ciencia. Inducción } \\
\text { a la metodología } \\
\text { de la ciencia }\end{array}$} & \multirow{3}{*}{$\begin{array}{l}\text { Comprende la } \\
\text { necesidad de realizar } \\
\text { observaciones } \\
\text { sistemáticas. }\end{array}$} & Comentar las respuestas. & Presencialmente \\
\hline & & & \\
\hline & & $\begin{array}{l}\text { Socialización en la plataforma de e- } \\
\text { aprendizaje }\end{array}$ & $\begin{array}{l}\text { Plataforma de e- } \\
\text { aprendizaje }\end{array}$ \\
\hline
\end{tabular}




\section{CONCLUSIONES}

Es evidente la falta de estrategias que favorezcan que los estudiantes interioricen los aprendizajes realizados, para que sean capaces de aplicarlos en diferentes contextos, en situaciones que requieran el uso integrado de los mismos. Es importante considerar una indagación en contextos reales. Estas estrategias deben tomar en cuenta la integración de elementos de la divulgación científica al inicio de las unidades didácticas, para ir avanzando en la formalización, mediante el uso de contextos cada vez más lejanos a la experiencia de los estudiantes, de manera que puedan irse apropiando de los saberes para que se logre la inculturación de la ciencia. La educación científica se debe concebir como un proceso de inmersión en las formas propias de proceder del ambiente científico, en la forma peculiar de ver las cosas característica de la escuela en la que se entronca. El diseño de estrategias centradas en el logro de competencias específicas se presenta como una alternativa para contribuir a la apropiación de la cultura científica.

\section{REFERENCIAS}

AAAS, Proyecto 2061 (documento en línea) http://www.project2061.org/esp/publications/ bsl/online/bolintro.htm. Acceso: 17 septiembre (2009).

Abrate, R; I., Gabetta y M. Pochulu La enseñanza de la Matemática en Ciencias Económicas ¿en contexto o fuera de contexto? Revista Iberoamericana de Educación Matemática, (12), 53-62 (2007).

Beneitone, P., y otros Reflexiones y perspectivas de la Educación Superior en América Latina. Informe final Proyecto Tuning América Latina 2004-2007. Univ. de Deusto. Bilbao, España (2007) [en línea]. http://tuning.unideusto.org/tuningal/index.php?option=com frontpage\&ltemid=1 Acceso: 6 de octubre de 2009

Camarena, G. P., Un enfoque de las Ciencias en Contexto desde la didáctica. Revista Innovación Educativa, 6(31), 21-31 (2006)

De Faria E., Control en la resolución de problemas. Cuadernos de investigación y formación en educación matemática. Universidad de Costa Rica. Asociación de Matemática Educativa, ASOMED. (2006). (en línea) http:///cimm.urc.ac.cr/ojs/index.php/CIFEM/article/VIEW/6/10. Acceso: 30 de enero (2010).

Eco U., La estructura ausente. Introducción a la semiótica. (3ª ed). España: Lumen (1986)

Flores, R; Torrado, C.; S. Mondragón y Pérez, C., Explorando la metacognición: Evidencia en actividades de lectura y escritura en niños y niñas de 5 a 10 años de edad. Revista Colombiana de Psicología, (12), 85-98. (2003).

Flores, J; Caballero, M.C. y Moreira, M.A., El laboratorio en la enseñanza de las ciencias: Una visión integral en este complejo ambiente de aprendizaje. Revista de Investigación № 68. Vol. 33 Septiembre- Diciembre 2009. [en línea]. Acceso 12 de febrero (2011).

Gil, D. y de Guzmán, M., Enseñanza de las ciencias y la matemática. Tendencias e innovaciones. Organización de los Estados Iberoamericanos para la Educación, la Ciencia y la Cultura. (1993). (en línea) http://www.oei.es/oeivirt/ciencias.pdf. Acceso: 4 de marzo (2009).

González, L.M. y Ruiz, E.F., Evaluación de competencias para la formación integral. Un análisis cualitativo. Memorias del IV Foro de Investigación educativa D.F., México. (2009).

González, L.M., Diseño de un plan de evaluación para la competencia investigativa en la formación de licenciados en Física. Memorias del $V$ Foro de Investigación Educativa. D.F. México. 27-28 pp. 29 - 33, mayo (2010).

González, L.M. y Rasilla, M., Una Estrategia para el Aprendizaje de la Cultura Científica. . Memorias del $\vee$ Congreso Internacional de Innovación Educativa. Merida, Yucatan, México 13-15 Pp. 120 - 125, octubre (2010).

Jorba, J; Gómez, I y Prat, A., Hablar y escribir para aprender. Síntesis Barcelona, España, (2000). 
Ladino, Y., Sobre la evaluación de competencias. Memorias del VI Congreso Internacional Investigación en la Didáctica de las Ciencias 12 al 15 de septiembre de 2001. p. 233-234 Barcelona: España, (2001).

Mayer, J .D., Salovey, P., Caruso, D.R. y Sitarenios, G., Emocional intelligence as a standard intelligence. Emotion, (1), pp. 232-242. (2001).

Nolasco, N y González, L.M., El Uso de la Pedagogía del Contrato en el Primer Curso de Laboratorio de Física de Nivel Superior en el Área de Ciencias. Memorias del V Congreso Internacional de Innovación Educativa. Merida, Yucatan, México 13-15 pp. 157 - 161, octubre (2010).

SEMS-SEP, Reforma integrar de la Educación Media Superior. (En línea): http://www.profordems.sems.gob.mx/profordems/ Acceso: 19 de diciembre (2009).

Sagan, C., La armonía de los mundos. En: Serie Cosmos, duración: 55 minutos (1979)

Sanmartí, N., Didáctica de las ciencias en la educación secundaria obligatoria. Barcelona, Ed. Síntesis S.A., España, 138-140 (2002).

Sebastián, J., La cooperación universitaria para el fomento de la cultura científica. Pensar Iberoamérica. Revista Pensar Iberoamérica, 8(4) s/p (2006) 